\title{
Probability Detection for Heterogeneous Networks Using FODPSO with ACO Optimization in Tunnel Based High Speed Trains
}

\author{
Priyanka $\mathrm{S}^{\mathrm{a}, 1}$, Leones Sherwin Vimalraj $\mathrm{S}^{\mathrm{b}}$, and Lydia $\mathrm{J}^{\mathrm{c}}$ \\ ${ }^{a} P G$ Scholar, Dept of ECE, pamimalar Engineering College, Chennai \\ ${ }^{b}$ Professor, Dept of ECE, Panimalar Engineering College, Chennai \\ ${ }^{c}$ Assistant Professor, Dept of EEE, Easwari Engineering College, Chennai
}

\begin{abstract}
Wireless communication on train is said to efficient when it has a good throughput and low latency. But the efficiency is reduced during underground tunnels and high speed trains. Heterogeneous network is said to be a network which is used to connect different kinds of network and hence it is said to be a efficient network. At times, it is found to be difficult to access the network. This problem can be overcome by using the optimization techniques such as FODPSO and ACO algorithm. It is proposed for optimizing the heterogeneous network by comparing different networks such as LTE, WiFi and WiMax. For efficient communication, the quality of services is important which should be maintained entire transmission.
\end{abstract}

Keywords. heterogeneousnetwork, ant colony optimization, long term evolution

\section{Introduction}

In recent years, vehicular communication network is increasingly popular in transport system. It provides small coverage for $\mathrm{WiFi}$ and WiMax for more kilometer coverage. When it is combined with $3 \mathrm{G}$ and WiMax it provides more security and high traffic performance. The algorithm specialty is to use ACO for optimizing and modifying membership function parameter. It helps to improve the quality of link network where it provides more reliability in network and improves efficiency of wireless network by combining various tests in different mobile scenarios[1].

\section{Related Works}

The IEEE 802.16 standard is used to provide seamless internet access named as WiMax which is the replacement of GSM and CDMA and provides comparatively more capacity with coverage of 1000 meters. It has 2-6 bands high speed mobility which is done in moving vehicle and MRS gain is provided for good data rate[2].In futuregeneration of heterogeneous network, Radio access technology is used for upcoming traffic demand. Parallel transmission is done for always best connection(ABC). Theheterogeneous network of $5 \mathrm{G}$ which is connected by multiple radio access technology to achieve more gain[3]. 
Vehicle to vehicle communication is only adapted in a low speed environment whereas in high speed surrounding it needs both $3 \mathrm{G}$ and WiMax for seamless communication. It is done by using MRS station network where it is said to be secure, real time and efficient [4]. Mobile relay station is used for communication between user terminal and base station. It is said to be efficient reliable and provides very good data transmission between user and base station. It is compared with three networks LTE, WiFi and WiMax with the user terminal and base station[5]. Ant colony algorithm is used for maximum utility in cognitive networks. The number of ants is used for to improve the performance of algorithm. The size of ants depends on performance of the system. When it has large ants it doesn't enhance the performance as the small ants[6]. A Switching Mechanism is needed to provide heterogeneous seamless connection. The technique used here is the ACO based on fuzzy logic algorithm. The main advantage is it has different MAC levels and high level mobility management protocol[7].

\section{Proposed Method}

\subsection{Ant Colony Optimization}

The ant colony optimization uses the ant colony system algorithm for the channel selection purpose. It is been inspired by the ants behavior in finding food. The ants search in very large distance and find foods by communication. It communicates with each other by a chemical substance named as phermone. By this process best nodes are selected and communication is been established [8].

The path selection is the path of the ants to reach the food. Firstly, ant selects the first node to select a particular channel where phermone is been applied. Then it goes to next node where the process is continued and by selecting the best nodes communication is established[9].

The path of ants is been calculated based on the probability function namely,

$$
P_{i j}^{k}=\left\{\begin{array}{c}
\arg \max (\tau(i, j)), \text { if } q \leq q_{0} \\
\frac{\tau(i, j)}{\sum \tau(i, s)}, \text { otherwise }
\end{array}\right.
$$

\subsection{FODPSO Algorithm}

The Fractional Order Dravian Particle Swarm Optimization is the optimization algorithm which is been derived by Particle Swarm Optimization. It adjust the nodes location in the search area with the neighboring particles. It is based on bird flocking where the bird find foods by flocking not by each individual. It calculates gbest and pbest which is reference node and selected node is compared and selects the best node[10]. In Figure 1, FODPSO algorithm it is found to be ellaborately explained in flowchart.

\section{Result And Discussion}

In Figure 2, The Detection Probability of Heterogeneous Network is said to be compared with LTE, WiMax and WiFi using FODPSO optimization. The Probability of detection is said to be 1 when it is efficient. As the system of detection probability is 
said to increase the SNR is also said to be increased. The SNR is said to be constant when there is no probability of detection. At a point when the probability detection is said to be established based on detection SNR also increases.

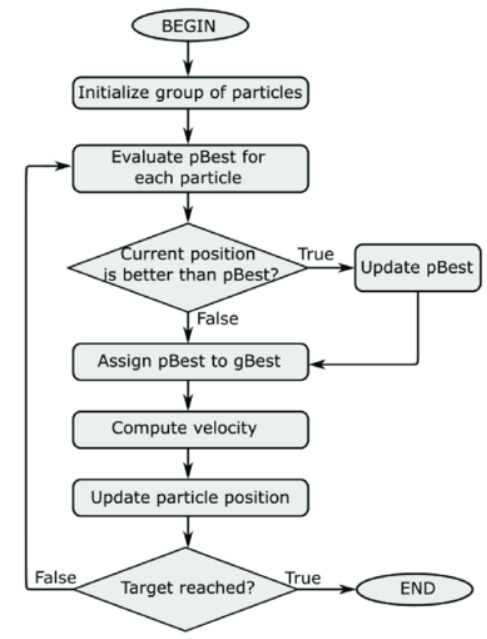

Figure 1. FODPSO algorithm flowchart

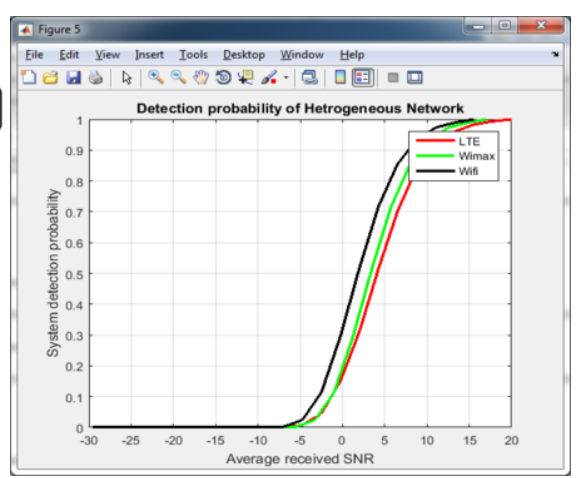

Figure 2. Detection probability of Heterogeneous Network

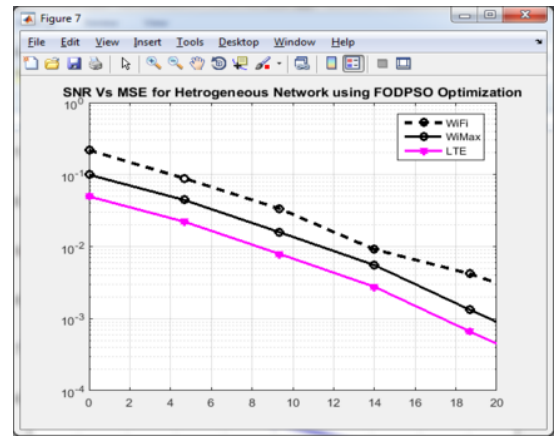

Figure 3. MIMO Channel Links Utility

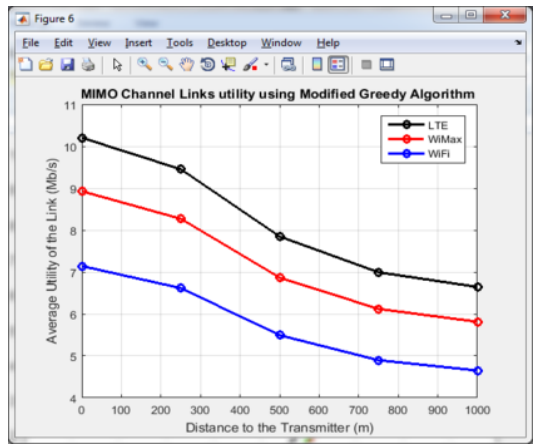

Figure 4. SNR Vs MSE using Modified Greedy Algorithm

In Figure 3, The MIMO channel link utility is found using the modified greedy algorithm which is been compared with LTE, WiMax and WiFi. The average utility of the link is been compared with the distance to the transmitter. As the distance is said to increase the average utility link is said to gradually decrease. For average $100 \mathrm{~m}$ the utility link is said to be veryefficient.

In Figure 4, The SNR Vs MSE for Heterogeneous Network using FODPSO optimization is been compared with LTE, WiMax and WiFi. It is found that error bellow $10^{\circ}$ is always negligible. As the MSE is said to increase the SNR is said to be decrease. 


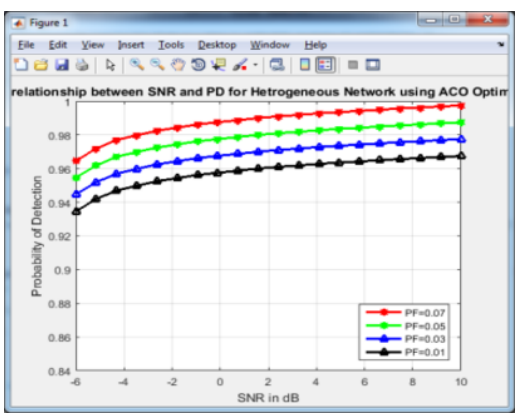

Figure 5. Throughput Maximization using FODPSO Optimization

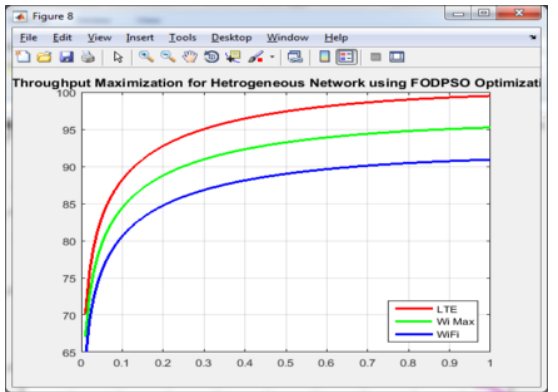

Figure 6. Relationship between SNR and PD using ACO Optimization

In Figure 5, The Throughput Maximization using FODPSO optimizationis compared with LTE, WiMax and WiFi. The Throughput is said to be efficient when it is said to be 1 . As the distance increases in metres the throughput is said to be maximum and efficient. The Throughput is said to be maximum inorder to check how efficient the data is been transmitted inrespect to time.

In Figure 6, The Relationship between SNR and PD using ACO Optimization is checked with each of the false alarm. The probability of detection and SNR is said to be compared. The probability of detection is said to be efficient when it is 1 . As the probability of detection is said to be increased then the SNR is also said to be increased.

In Figure 7, The Convergence Plot is found using ACO optimization and FODPSO optimization which is compared with LTE, WiMax and WiFi. For FODPSO and ACO optimization it is found that as the sum rate that is frequency increases with the distance in metres. In which the LTE is found to be efficient with nearly 40bits per $\mathrm{Hz}$ than the other networks such as WiMax and WiFi for FODPSO optimization.

For ACO optimization it is found that LTE is found to be efficient with nearly 50bits per $\mathrm{Hz}$ than the other networks such as WiMax and WiFi for ACO optimization.

In Figure 8, Optimization of Link quality is compared with ACO and FODPSO optimization. The Link quality Strength is been compared with the sumrate in bitz per Hz. As the Link quality is said to be increased the sumratethat is frequency also increases. As a result it is found that FODPSO optimization has the frequency nearly 50 bits per $\mathrm{Hz}$ whereas ACO optimization has the frequency above 60bits per $\mathrm{Hz}$ which is more efficient.

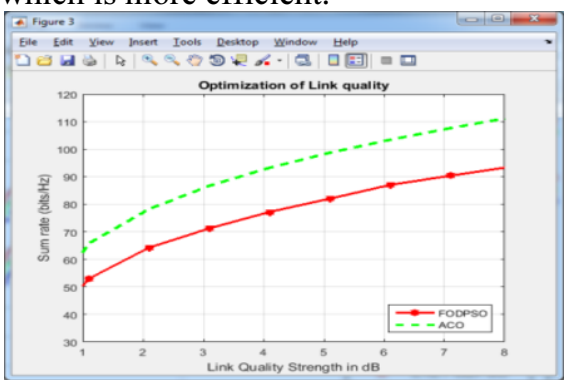

Figure 7. Convergence Plot

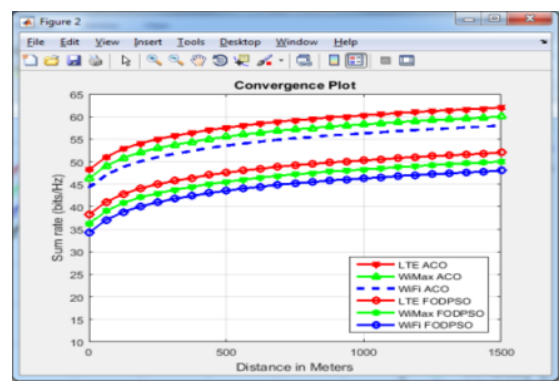

Figure 8. Optimization of Link Quality 


\section{Conclusion}

In high speed mobile scenario tests are conducted to research the efficiency of heterogeneous networks. The proposed FODPSO and ACO optimization produces high efficiency, throughput and utility links. Firstly, Wireless network characteristics of various nodes are calculated. Secondly, advantage of profiting from various nodes are analyzed and algorithm may also be used to increase the reliability in another multipath schedule algorithm[11].

\section{References}

[1] Jiyan Wu, Member, Ieee, Chau Yuen, Bandwidth-Efficient Multipath Transport Protocol For QualityGuaranteed Real-Time Video Over Heterogeneous Wireless Networks. IEEE Transactions On Communications, Vol. 64, No. 6, June 2016.

[2] Mohamed M. E. A. Mahmoud1, Xiaodong, Secure And Reliable Routing Protocols For Heterogeneous Multihop Wireless Networks.1045-921, Ieee Transactions On Parallel And Distributed Systems, Volume: 26, Issue: 4 , April 2015.

[3] Ali Al-Sherbaz, Sabah Jassim,Private Synchronization Technique For Heterogeneous Wireless Network (Wifi And Wimax),Proceedings Of Spie - The International Society For Optical Engineering, April 2008 Doi: $10.1117 / 12.777580$

[4] Abhijit Sarma, Member, Ieee, Sandip Chakraborty, Deciding Handover Points Based On Context Aware Load Balancing In A Wifi-Wimax Heterogeneous Network Environment,IEEE Transactions On Vehicular Technology, Vol. 65, No. 1,Pp No 348-358, January 2016.

[5] Duc Chinh Hoang, Real-Time Implementation Of A Harmony Search Algorithm-Based Clustering Protocol For Energy-Efficient Wireless Sensor Networks, IEEE Transactions On Industrial Informatics, Vol. 10, No. 1,February 2014.

[6] Yan Jiao And Inwhee Joe, Energy-Efficient Resource Allocation For Heterogeneous Cognitive Radio Network Based On Two- Tier Crossover Genetic Algorithm.Journal Of Communications And Networks, Vol. 18, No. 1, February 2016

[7] Yongjun Sun, Wenxin Dong And Yahuan Chen, An Improved Routing Algorithm Based On Ant Colony Optimization In Wireless Sensor Networks,. IEEE Communications Letters, Vol. 21, No. 6, Pp No 13171321, June 2017

[8] Rejina Parvin,Vasanthanayaki ;Particle Swarm Optimization Based Clustering By Preventing Residual Nodes In Wirelesssensor Networks; 1530-4376, Ieee Sensors Journal, Volume: 15, Issue: 8 , Aug. 2015.

[9] S. Priyanka, Dr S. Leones Sherwin Vimalraj, J.Lydia, Seamless Communication Network For Enhanced Performance In Tunnel Based High Speed Trains Using Q-Leach And Fodpso Algorithm,International Journal Of Innovative Technology AndExploring Engineering (Ijitee) Issn: 2278-3075, Volume-9, Issue1s, November 2019.

[10] S. Priyanka, Dr S. Leones Sherwin Vimalraj, J.Lydia ,Seamless Communication Models For Enhanced Performance In Tunnel Based High Speed Trains Springer Nature Switzerland Ag 2020 Emerging Trends In Computing And Expert Technology, Comet 2019, Lndect 35, Pp. 1580-1591,January 2020

[11] S. Leones Sherwin Vimalraj, V. Kannan, S. Ravi And M. Anand, Modulation Identification And Hamming Encoding Technique Implemented In Software-Defined Radio.American Journal Of Applied Sciences 11 (4): 656-665, 2014, Issn: 1546-9239 C2014 Science Publication. 